\title{
Lattice-Boltzmann simulation of the sedimentation of charged disks
}

\author{
Fabrizio Capuani \\ FOM Institute for Atomic and Molecular Physics (AMOLF), Kruislaan 407, 1098 SJ Amsterdam, \\ The Netherlands \\ Ignacio Pagonabarraga ${ }^{\text {a) }}$ \\ Departament de Física Fonamental, C. Martí i Franqués 1, 08028 Barcelona, Spain \\ Daan Frenkel ${ }^{\text {b) }}$ \\ FOM Institute for Atomic and Molecular Physics (AMOLF), Kruislaan 407, 1098 SJ Amsterdam, \\ The Netherlands
}

(Received 22 June 2005; accepted 20 January 2006; published online 24 March 2006)

\begin{abstract}
We report a series of lattice-Boltzmann simulations of the sedimentation velocity of charged disks. In these simulations, we explicitly account for the hydrodynamic and electrostatic forces on disks and on their electrical double layer. By comparing our results with those for spheres with equal surface and charge, we can clarify the effect of the particle shape on the sedimentation process. We find that disks and spheres exhibit a different dependence of the sedimentation velocity on the Debye screening length. An analysis of the behavior of highly charged disks (beyond the scope of the linearized Poisson-Boltzmann equation) shows that, in that regime, the charge dependence of the sedimentation velocity of disks and spheres is similar. This suggests that, at high charge, the effective hydrodynamic shape of the disks becomes more spherical. (C) 2006 American Institute of Physics. [DOI: 10.1063/1.2178804]
\end{abstract}

\section{INTRODUCTION}

Suspensions of charged disks are of great practical importance. Examples range from clay suspensions to biological fluids. In the present paper, we present calculations of the electrokinetic behavior of charged disks. As disks are not spherically symmetric, they also provide an ideal model system to study the effect of shape on the coupling between electrostatic and hydrodynamic responses of a macroscopic particle.

There exists experimental interest and knowledge in the transport properties of suspensions of charged disks. ${ }^{1}$ Yet, in spite of the importance of these systems, there is surprisingly little theoretical knowledge about the effect of the charge of the disks on their transport properties. One reason may be that the nonspherical geometry greatly complicates the use of the analytical approach that is used to describe charged spherical particles. While there are papers that consider the hydrodynamical properties of uncharged disks ${ }^{2}$ or the electrostatic properties of charged disks, ${ }^{3,4}$ we are not aware of any theoretical publications that treat the interplay between electrostatics and hydrodynamics for charged disks.

Several theoretical studies suggest that, in general, there may be nontrivial coupling effects due to shape asymmetries. ${ }^{5,6}$ The need for a numerical (rather than an analytical) approach is related to the fact that the analytical approaches are usually only tractable in certain simplified limits. For example, to arrive at tractable analytical expressions it is often necessary to assume that the Poisson-Boltzmann equation can be linearized or that the Debye screening length

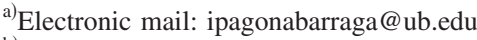

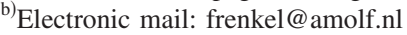

is small compared to the linear dimensions of the charged colloid. Yet these conditions are rarely satisfied for charged biocolloids and biopolymers. These (nonspherical) particles usually have high charges under physiological conditions, making the use of the linearized Poisson-Boltzmann equation questionable.

The total number of ionizable groups of colloids can be determined experimentally by titration. However, the experimental determination of the effective charge of colloids usually exploits nonequilibrium techniques, such as electrophoresis or sedimentation. The interpretation of many of these experiments is based on theoretical expressions that have been derived for weakly charged spherical colloids. Yet, it is not at all obvious that expressions valid for spheres can be extrapolated to disks. Hence, it is important to understand the effect of shape and charge on the transport behavior of nonspherical charged particles.

In the present paper we report a numerical study of the electrokinetics of charged disks, using a kinetic lattice model based on the Boltzmann equation. Different implementations of electrokinetic phenomena have been proposed within a lattice-Boltzmann (LB) scheme $^{7-10}$ and finite-difference methods. ${ }^{11}$ We use a LB method introduced previously ${ }^{12}$ and which has been shown to perform well when coupling charged solid particles to solvent flows. As any other LB scheme, this method ignores charge fluctuations and static charge correlation effects, and it allows the study of charged particles' dynamics at the nonlinear Poisson-Boltzmann level.

Our simulations extend from the regime where the electrohydrodynamic coupling is small (low charge, thin double layer) to the case where the charge is large and the double 
layer is extended. By performing simulations of many different conditions, we can disentangle the effects of charge, double layer width, and shape.

The paper is organized as follows. In Sec. II we present the basic electrokinetic equations and the simulation method that we use to solve them. In order to study the effect of charge on the sedimentation of disks, we must first know the sedimentation behavior of uncharged disks. This is done in Sec. III, where we use our model to compute the friction coefficients of a neutral sedimenting disk for motions parallel to and perpendicular to its axis of symmetry (we refer to these motions as "longitudinal" and "transverse," respectively). In Sec. IV we discuss the effect of the charge of the disk on the sedimentation velocity. In Sec. V we consider the effect of the concentration of the disks on the sedimentation velocity. Section VI focuses on the variation of the sedimentation velocity with Debye screening length. Section VII contains a comparison of the sedimentation behavior of disks and spheres. Concluding remarks are contained in Sec. VIII.

\section{ELECTROKINETIC MODEL}

We analyze a simple geometry in which one disklike colloidal particle with radius $a$ and height $h$ sediments due to the action of a uniform external field. The disk has an overall charge $Q=Z e$, where $Z$ is the valency and $e$ is the elementary charge unit. The aspect ratio $p$ is defined as $p=2 a / h$. The disk is suspended in a symmetric electrolyte and, for the sake of simplicity, we assume that coions and counterions have the same mobility. The fluid mixture is characterized locally by the solvent density $\rho_{s}$ and by the microion electrolyte densities $\rho_{ \pm}$. The latter also determine the local charge density of the fluid, $q(\mathbf{r})=z e\left[\rho_{+}(\mathbf{r})-\rho_{-}(\mathbf{r})\right]$. We restrict ourselves to monovalent electrolytes, i.e., $z=1$.

On a macroscopic length scale, the dynamics of the system is governed by the standard electrokinetic equations ${ }^{13}$ that specify the interplay between the electrical potential, local charge density, electrical currents, and fluid flow:

$$
\begin{aligned}
& \frac{\partial}{\partial t} \rho_{k}=-\nabla \cdot \mathbf{j}_{k}, \quad k=+,-, \\
& \frac{d}{d t}(\rho \mathbf{v})=\eta \nabla^{2}(\rho \mathbf{v})-\nabla P+\frac{k_{B} T}{e} q \nabla \Phi, \\
& \mathbf{j}_{k}=-\rho_{k} \mathbf{v}+D_{k}\left[\nabla \rho_{k}+z_{k} \rho_{k} \nabla \Phi\right],
\end{aligned}
$$

where $\eta$ is the shear viscosity, $P$ is the pressure, $\mathbf{v}$ is the fluid velocity, $k_{B} T \equiv \beta^{-1}$ measures the temperature, and $D_{k}$ stands for the diffusivity of each electrolyte species (which reduce to a single constant for symmetric electrolytes). $\hat{\Phi}$ is the electrostatic potential, while $\Phi \equiv \hat{\Phi}\left(k_{B} T / e\right)$ is an appropriate dimensionless potential which satisfies the Poisson equation

$$
\nabla^{2} \Phi=-4 \pi l_{B}\left[\sum_{k= \pm} z_{k} \rho_{k}+\rho_{w}\right],
$$

where $l_{B}=\beta e^{2} /(4 \pi \epsilon)$ is the Bjerrum length and $\epsilon=\epsilon_{0} \epsilon_{r}$ denotes the dielectric constant of the medium, while $\rho_{w}$ refers to the charge density due to embedded solid objects, either colloids of solid walls.
Equation (1) simply expresses a conservation law and Eq. (3) is the constitutive equation. Together with the incompressibility condition $\nabla \cdot \mathbf{v}=0$, Eq. (2) corresponds to the Navier-Stokes equations for an incompressible, isothermal electrolyte. In the presence of external forces (such as the gravitational field), the corresponding force must be added to the right-hand side of Eq. (2).

\section{A. Simulation method}

To simulate the sedimentation of charged disks, we used the lattice-Boltzmann scheme reported in Ref. 12. We showed therein that lattice Boltzmann can be used to compute transport coefficients of charged spherical colloids. Below, we briefly summarize the main features of the method and refer the reader to that reference for further details.

The LB method is the lattice counterpart of the Boltzmann equation. It prescribes a dynamical evolution rule for the distribution function $n_{i}(\mathbf{r}, t)$, which represents the density of particles at the lattice node $\mathbf{r}$, at the discrete time $t$ and with the discrete velocity $\mathbf{c}_{i}$. The density-weighted moments of the local velocity distribution correspond to the hydrodynamic fields. In particular, $\Sigma_{i} \mathbf{c}_{i} n_{i}(\mathbf{r}, t)=\rho \mathbf{v}$ is the fluid's momentum; it satisfies the Navier-Stokes equation on length and time scales that are large compared to the lattice spacing and the LB time step, respectively. The use of LB avoids having to solve for the pressure explicitly. Moreover, it is easy to deal with general geometries. ${ }^{14}$ Since LB is a kinetic model, it provides a framework where it is easier to introduce new features from a physically motivated perspective.

The electrolyte species are simulated by following the diffusion and convection of the local densities of coions and counterions described by Eqs. (1) and (3). This equation is based on the flux of each species along the links that connect neighboring nodes and ensures strict local charge conservation. This local charge combined with the corresponding electrostatic potential — computed by a numerical solution of the Poisson equation (4) - provides the local force that accelerates the fluid. With this technique, colloidal particles are simply introduced as surfaces where the collision rules of the populations of the neighboring nodes are modified to ensure nonslip boundary conditions. ${ }^{15}$ The link-based definition of the flux of the electrolyte species leads to a straightforward implementation of the no-flux boundary condition for each of the ionic species at solid surfaces. This suppresses possible charge leakage through the solid walls.

In Ref. 12 we checked systematically how discretization affects electrokinetics by comparing the simulated results with existing theoretical predictions, where available. We found excellent agreement; the only numerical limitation being the need to resolve properly the double layer width, which becomes more costly as a finer grid is required when decreasing the double layer if the numerical accuracy is kept constant.

For computational convenience, we choose the value of the kinematic viscosity $\nu=1 / 6$ [in lattice units (l.u.)] (Ref. 15) and the $\rho_{s}=1$ l.u., as the density unity. The Boltzmann temperature $k_{B} T=1 / 2$ l.u. to ensure thermodynamic consistency (see Ref. 12 for details). The external (gravitational) 
field that induces sedimentation was chosen to be $10^{-6}$, a value that is well inside the linear-response regime. This gravitational field generates fluid velocities of the order of $10^{-8}$ l.u. This choice of the gravitational field ensures that all our simulations are free from nonlinear effects, while numerical round-off errors are absent. The diffusivity of the electrolyte is set to $D=0.19$, a value for which spurious diffusion due to lattice advection is negligible (see Ref. 12). When expressed in the appropriate units for aqueous electrolyte solutions, the diffusion coefficient chosen is typically one order of magnitude smaller than that of small salt ions. In the simulations described in the subsequent sections we vary the salt concentration between $7 \times 10^{-4}$ and $5 \times 10^{-3}$ as a way to control the electrical double layer thickness and its effect on sedimentation so that $\kappa R$ ranges from values smaller than unity to values larger than unity.

In all the simulations the Bjerrum length $l_{B}=0.4$, ensuring that it is always smaller than the Debye length. For water at room temperature, $l_{B}=7 \AA$ which implies that our simulations correspond to colloids of nanometric size. Both Reynolds and electrolyte Péclet numbers are much smaller than unity in the simulations described below, ensuring a realistic setting of dimensionless numbers (see, e.g., Ref. 16 for a discussion on the relevance of dimensionless numbers in LB). In our simulations the diffusion coefficient has a value which corresponds to nanometric electrolytes (again for monovalent ions in water). While in principle we can access any value of the diffusion coefficient, using larger values for $D$ results in a drastic slowing down of the simulations. Although the diffusion coefficients that we use are unrealistic for simple salts, they can be relevant for nonaqueous solutions of colloidal disks or aqueous solutions of colloidal disks with large, weakly charged, molecular counterions. Finally, the surface charge densities used in the simulations are smaller than 1 in units of $e / l_{B}^{2}$; hence neglecting electrostatic correlations is a consistent approximation [in fact, since $R / l_{B} \sim 10, Q /\left(4 \pi R^{2}\right) \sim Z \times 10^{-3}$ in units of $\left.e / l_{B}^{2}\right]$.

\section{SEDIMENTATION OF NEUTRAL DISKS}

Before assessing the role of electrostatics on the sedimentation of nonspherical particles, we performed LB simulations to compute the sedimentation velocity of uncharged hard disks. Such reference calculations are needed because, in contrast to the case of hard spheres, analytic expressions for the sedimentation velocity of an isolated hard disk only exist in the limit of infinitely thin disks. ${ }^{17,18}$ We are not aware of analytical results for disks with finite aspect ratios.

We have simulated the sedimentation of disks with two different nominal aspect ratios $p=10$ and $p=5$, corresponding to disks of lateral dimension $h=2$, and radii $a=10$ and 5, respectively. However, these aspect ratios are only approximate: in the LB approach, the hydrodynamic boundary of a solid particle is usually located close to the midpoint of links joining fluid and solid nodes. In practice, the hydrodynamic shape of an object may differ slightly from the nominal one. For this reason, we need to calibrate the shapes of the disks. An unambiguous way to determine these effective sizes (the hydrodynamic radius and height) would be to measure the

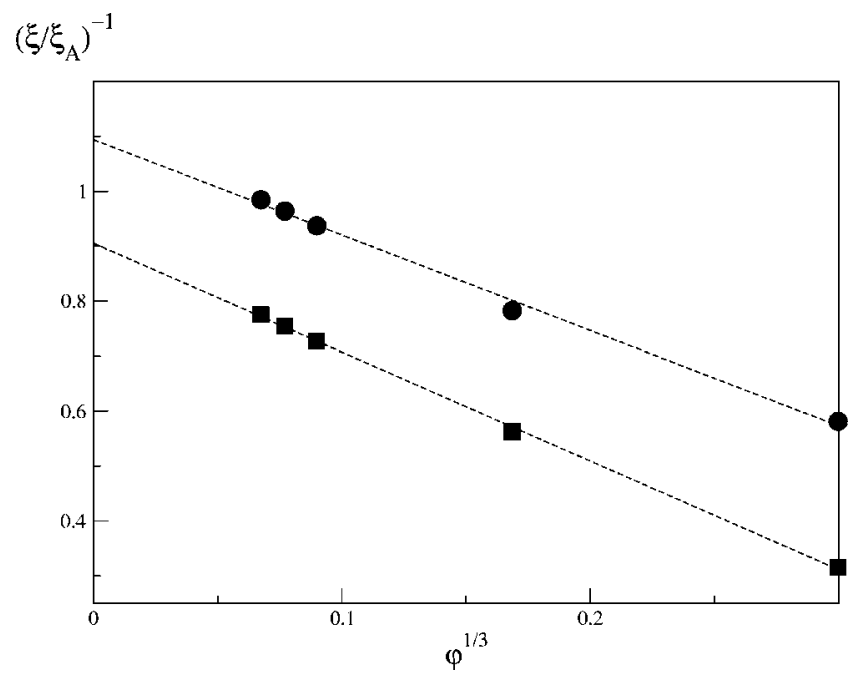

FIG. 1. Transverse (circles) and longitudinal (squares) friction coefficients for a sedimenting neutral disk of aspect ratio $p=5$ as a function of the volume fraction of the array. The friction coefficients are normalized by the friction coefficient of a sphere with the same radius as the cylinder, i.e., $\xi_{A}=6 \pi \eta R$. The dashed lines are linear fits.

friction coefficients of the particles and compare the results with the appropriate analytical expression of an infinitely thin disk. Great care must be taken when comparing the LB numerical results for the friction coefficient with results obtained for an isolated disk. Since we use periodic boundary conditions, the simulations measure the friction coefficient of a regular array of particles at volume fraction $\varphi=\pi a^{2} h / L^{3}$, where $L$ is the diameter of the simulation box. Hence, in order to extrapolate to infinite dilution, we must perform a series of simulations with increasing box size. The same procedure will also be used to compute the sedimentation velocity of a charged disk in the dilute limit.

\section{A. Friction coefficients}

In the following calculations, the reference frame is centered on the disk and the disk is fixed on the lattice. Hence, the gravitational force acting on the disk becomes a body force, with opposite direction, that acts to the fluid. We then determined the total fluid velocity at steady state $v_{F}$. As this velocity is equal and opposite to the sedimentation velocity of the particle, $U_{d}=-v_{F}$, we can relate the sedimentation velocity to the friction coefficient through $U_{d}=F_{g} / \xi$, where $U_{d}$ is the sedimentation velocity of the disk, $\xi$ is its friction coefficient, and $F_{g}$ is the applied gravitational force. We computed both the friction coefficient for motion along the symmetry axis of the disk and the one for perpendicular (transverse) motion.

Hashimoto ${ }^{19}$ has shown that the friction coefficient of an array of hard spheres depends, at low volume fractions $\varphi$, as $\left(\xi / \xi_{0}\right)^{-1}=1-1.76 \varphi^{1 / 3}+\varphi+O\left(\varphi^{2}\right)$, which shows that the initial decrease in the sedimentation velocity of an array of hard spheres is controlled by the colloid volume fraction.

In Fig. 1 we show the transverse and longitudinal friction coefficients for a neutral disk with $p=5$ at various volume fractions, normalized by the Stokes friction coefficient of a sphere with the same area, i.e., by $\xi_{A} \equiv 6 \pi \eta R$, 
TABLE I. Friction coefficients of an isolated disk in transverse and in longitudinal motions normalized by the Stokes friction coefficient $\xi_{A}=6 \pi \eta R$ of a sphere with equal surface area, i.e., with radius $R=\sqrt{a(a+h) / 2}(a$ is the radius of the cylinder). We compare the computed value of the friction coefficients with approximate theoretical values for an oblate spheroid with the two axes equal to the disk radius and to the disk height. We studied two disks with aspect ratios $p=5$ and $p=10$, respectively.

\begin{tabular}{lccccc}
\hline \hline & \multicolumn{2}{c}{$p=5$} & & \multicolumn{2}{c}{$p=10$} \\
\cline { 2 - 3 } \cline { 5 - 5 }$\xi_{A}=6 \pi \eta R$ & Simulation & Oblate spheroid & Simulation & Oblate spheroid \\
\hline$\xi_{\perp} / \xi_{A}$ & 0.91 & 0.9 & & 0.93 & 0.85 \\
$\xi_{\|} / \xi_{A}$ & 1.1 & 1.06 & & 1.19 & 1.11 \\
$\xi_{\perp} / \xi_{\|}$ & 0.83 & 0.84 & 0.78 & 0.77 \\
\hline \hline
\end{tabular}

$R=\sqrt{a(a+h) / 2}$. The figure shows that, just as in the case of neutral spheres, the friction varies linearly with $\varphi^{1 / 3}$. Knowledge of this concentration dependence allows us to extrapolate the numerical results to estimate the friction coefficients $\left(\xi_{\perp}\right.$ and $\left.\xi_{\|}\right)$at infinite dilution. One limiting case is known: the friction coefficients of infinitely thin disks $(p \rightarrow \infty)$ are identical to that of an oblate spheroid with the same aspect ratios between its main axis. ${ }^{18}$

We summarize the results for the normalized friction coefficients for disks of two aspect ratios in Table I. For the disk with $p=5$, we conclude that, to a good approximation, the hydrodynamic radius and height correspond to the nominal ones. The numerical values obtained for the larger disk $(p=10)$ are less satisfactory than for the shorter one. While one could conclude that, for a larger aspect ratio, the hydrodynamic radius and height are different with respect to the nominal ones, and hence recompute the effective values for $a$ and $h$ so as to adjust $\xi_{A}$, this does not seem satisfactory, since for a larger object the disagreement between the nominal and hydrodynamic sizes is expected to decrease. Moreover, because we have always used cubic simulation boxes, we can attribute such a difference to the stronger coupling between image disks for longitudinal sedimentation. On the other hand, the ratio between the perpendicular and parallel frictions $\xi_{\perp} / \xi_{\|}$do agree with the value estimated on the basis of the nominal size, suggesting that the deviations come mostly from uncertainties related to the extrapolation from finite volume fraction values. Hence, we conclude that, also for this shape, the disagreement between the two sizes is negligible, and we ascribe the deviations to interactions with the periodic images.

\section{SEDIMENTATION VELOCITIES OF CHARGED DISKS: CHARGE DEPENDENCE}

Before discussing the computed sedimentation velocities of charged disks, we briefly recall the theoretical results concerning the sedimentation velocity of weakly charged spheres, since this theory serves as a reference point for the discussion of the results for disks. Booth ${ }^{20}$ (and Ohshima et $a l^{21}$ ) predicted that the sedimentation velocity $U_{s}(Z)$, where $Z$ is the valence, of an isolated sphere is a quadratic function of the sphere valence, which can be expressed as

$$
\frac{U_{s}(Z)}{U_{s}(0)}=1-c_{2}(\kappa R) Z^{2},
$$

where $U_{s}(0)$ corresponds to the sedimentation velocity of a hard sphere in the dilute limit. In the regime where the Debye-Hückel theory is valid, the prefactor $c_{2}$ for a symmetric 1-1 electrolyte is given by

$$
c_{2}(\kappa R)=\frac{k_{B} T l_{B}}{72 \pi R^{2} \eta D} f(\kappa R),
$$

where $R$ is the radius of the sphere and, with $\rho_{k}^{0}$ denoting the bulk value of the charge density of species $k, \kappa$ $=\sqrt{4 \pi l_{B} \sum_{k} z_{k}^{2} \rho_{k}^{0}}$ stands for the inverse Debye length that characterizes the size of the electrical double layer. The function $f(\kappa R)$ is defined as

$$
\begin{aligned}
f(\kappa R)= & \frac{1}{1+(\kappa R)^{2}}\left[\mathrm{e}^{2 \kappa R}\left(3 E_{4}(\kappa R)-5 E_{6}(\kappa R)\right)^{2}\right. \\
& +8 \mathrm{e}^{\kappa R}\left(E_{3}(\kappa R)-E_{5}(\kappa R)\right)-\mathrm{e}^{2 \kappa R}\left(4 E_{3}(2 \kappa R)\right. \\
& \left.\left.+3 E_{4}(2 \kappa R)-7 E_{8}(2 \kappa R)\right)\right],
\end{aligned}
$$

expressed as a linear combination of the integral function, $E_{n}(x) \equiv x^{n-1} \int_{x}^{\infty} d t t^{-n} \exp (-t)$. Since the values for the diffusion $D$ we are using are smaller than those characteristic to molecular electrolytes, we will obtain a larger reduction of sedimentation velocities. In the linear regime, such a change only modifies the magnitude but not the trends we will discuss, as tested quantitatively in Ref. 12. In the nonlinear regime we have seen that changing $D$ does not modify significantly the qualitative dependence of the sedimentation velocity, even if the simple proportionality is lost. If we assume that the diffusion coefficient follows the StokesEinstein relation, $D=k_{B} T /\left(6 \pi \eta a_{s}\right)$ (with $a_{s}$ being the electrolyte size), then Eq. (6) can be expressed as $c_{s}$ $=\left(l_{B} a_{s} / R^{2}\right) f(\kappa R) / 12$, making explicit the role that molecular size plays in the magnitude of the sedimentation velocity.

Equation (7) provides the general features of the shape of the sedimentation curves as function of $k R$ ( $\kappa a$ when the curves refer to disks) depicted in Figs. 5-8.

It is reasonable to assume that, in the case of disks, the dependence of the sedimentation velocity on colloidal charge in the Debye-Hückel limit has the same functional form as Eq. 5, where all the shape dependence and hydrodynamic coupling enter through the factor $c_{2}(\kappa R)$. To test this and to analyze the role of charge on the sedimentation velocity of disks, we performed a series of simulations at constant Debye screening length and volume fraction $\left(\varphi=7.2 \times 10^{-4}\right.$ for $p=5$ and $\varphi=2.9 \times 10^{-3}$ for $\left.p=10\right)$. Since in many cases of practical interest colloidal particles are highly charged (see e.g., Ref. 22 for the case of disklike clay particles) we performed numerical simulations covering a wide range of disk charges.

The results obtained are displayed in Fig. 2, where the velocity, normalized by the sedimentation velocity of a hard disk at the same volume fraction, is depicted as a function of 


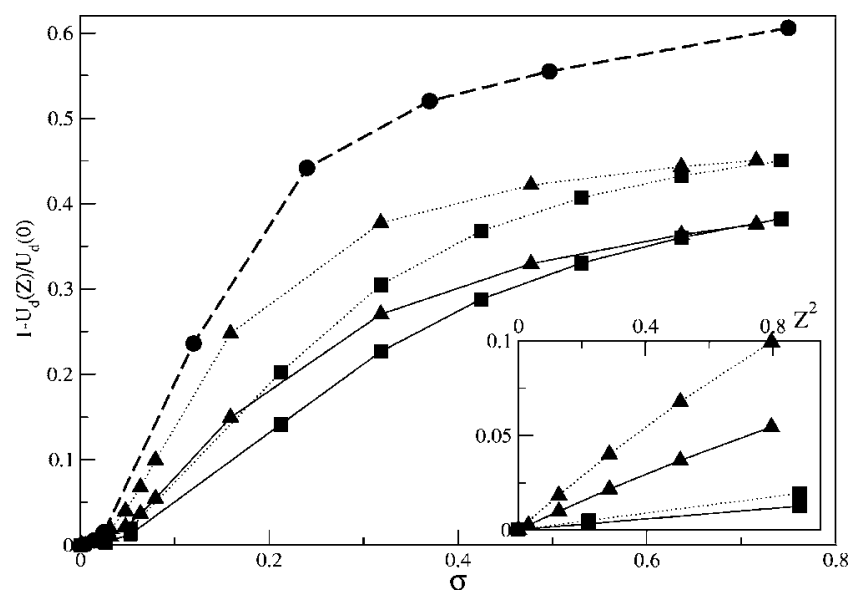

FIG. 2. Surface-charge-density dependence of $1-U_{d}(Z) / U_{d}(0)$ for a disk with aspect ratios $p=5$ (squares) and $p=10$ (triangles) in transverse (dotted lines) and longitudinal (continuum lines) motions at $k a=1$. For comparison, we also show the behavior for a sphere with radius $R=4.2$ (circles). In the inset, we show $1-U_{d}(Z) / U_{d}(0)$ for weakly charged disks as a function of $Z^{2}$. The curves for the disk with $p=10$ are plotted against $Z^{2} / 10$ to show them on the same scale. The inset shows that for small charges, the sedimentation velocity varies quadratically with charge. The lines in the inset are linear fits to the simulation data. In contrast, the curves in the main figure are simply meant as a guide to the eye.

the surface charge $\sigma=e Z /[2 \pi a(a+h)]$. One can identify the quadratic dependence at low charge, consistent with Booth theory for spheres. Such dependence can be clearly appreciated in the figure's inset. After this quadratic growth, a crossover region is identified, for surface charge densities between 0.1 and 0.4 , before entering the asymptotic regime at even larger surface charge densities, where the sedimentation velocity increases much more slowly. This behavior is consistent with numerical results on the sedimentation velocity of charged colloidal spheres which also show a deviation from Booth's predictions for surface charge densities around 0.1 .

Comparing the deviation of the sedimentation velocity for transverse and longitudinal motions, as displayed in Fig. 2, one can see that the sedimentation velocity decreases faster with charge for transverse motion, regardless of the aspect ratio. This is indicative of a stronger electrokinetic coupling for transverse motion. Interestingly, the sedimentation velocities [expressed by $1-U_{d}(Z) / U_{d}(0)$ ] become almost independent of the aspect ratio of the disks for large surface charge densities. In the same figure, we also plot the decrease in sedimentation velocity for a sphere of radius $R$ $=4.2$, which shows the same dependence on surface charge as that of the disks.

Figure 2 allows us to draw some qualitative conclusions concerning the nature of the errors that are made when estimating the charge of disklike particles by assuming the validity of Booth's theory. ${ }^{22}$ As mentioned above, Booth's theory is valid only for weakly charged sedimenting spheres. As we now have numerical results for the sedimentation velocity of disks, we can identify two sources of errors. At low charge, where the quadratic dependence of the sedimentation velocity on colloidal charge holds, there will still be some discrepancy in the coefficient $c_{2}$. We address this issue in more detail in Sec. VII. At high charge, where even the quadratic surface charge dependence does not apply, the use of

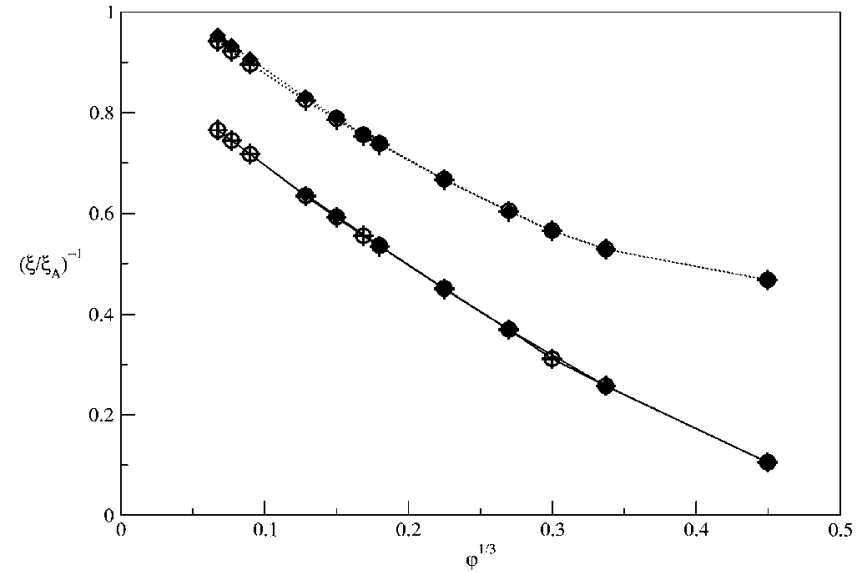

FIG. 3. Volume-fraction-dependent normalized friction coefficients for a disk with radius $a=5$, aspect ratio $p=5$, and valency $Z=10$ for $\kappa a$ equal to 0.5 (spheres), 0.8 (pluses), and 2.1 (filled diamonds). The upper curves are for the transverse friction coefficient, while the lower curves are for a longitudinal friction coefficient. The curves are normalized by the friction coefficient of an isolated sphere with equal surface area $\xi_{A}=6 \pi \eta \sqrt{a(a+h) / 2}$ (see Table I for the corresponding neutral values). Curves are drawn as a guide to the eye.

the Booth theory leads to serious errors in the estimation of the zeta potential. To give an idea of the magnitude of this error, one should compare the curves shown in Fig. 2 with a parabolic extrapolation of the curves up to $\sigma \simeq 0.1$. Our calculations also suggest that if the sedimentation velocity of a charged colloid is plotted as a function of the surface charge density (and not as a function of the total charge as one might be tempted to do when comparing with Booth's theory) the curves corresponding to the sedimentation velocity of a variety of charged disks show the same functional dependence.

\section{SEDIMENTATION VELOCITIES OF CHARGED DISKS: VOLUME FRACTION DEPENDENCE}

As discussed above, the inverse friction coefficient of a dilute, ordered array of hard spheres scales as $\varphi^{1 / 3}$. In Ref. 12, we have verified that this functional dependence also holds for charged spheres, provided that the system is dilute enough to guarantee that there is no significant overlap of the double layers. For charged disks, we expect the $\varphi^{1 / 3}$ dependence to hold under the same circumstances. In order to test whether there is a detectable effect of the overlap of electric double layers of different disks, we have computed the normalized friction coefficients for disks of aspect ratio $p=5$ as a function of the volume fraction, for volume fractions up to $10 \%$, for different widths of the diffuse layer.

In Fig. 3 we show the results for a weakly charged disk both for transverse and longitudinal motions. Note that, in the dilute limit, the friction coefficients will depend on $\kappa a$ due to the electrohydrodynamic interaction. For transverse motion the convergence to the dilute limit is slower, indicating a stronger coupling between disks; we attribute this to the fact that the distance of closest approach coincides with the external field direction. Although for $\kappa a=1 / 2$ and high volume fractions the diffuse layers overlap, the effect of the diffuse layer is much weaker than the volume fraction depen- 


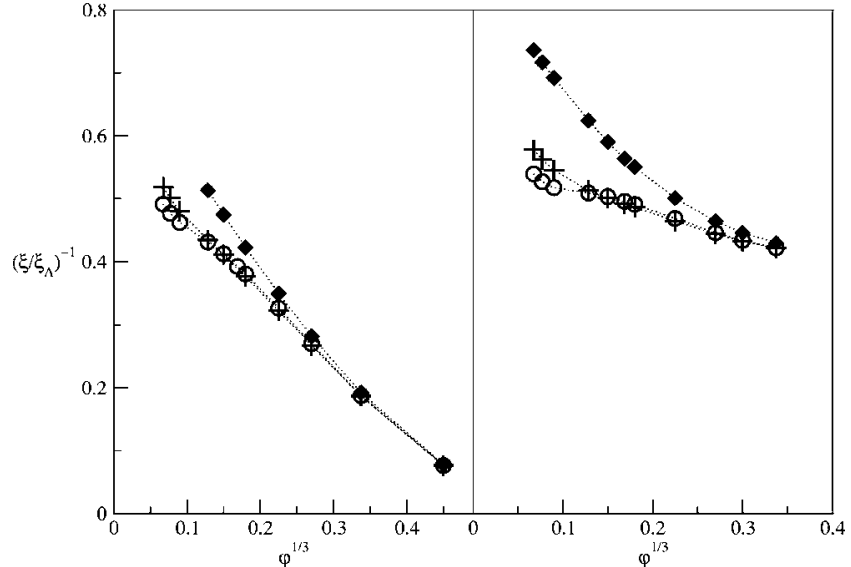

FIG. 4. Volume-fraction-dependent normalized friction coefficients for a disk with radius $a=5$, aspect ratio $p=5$, and valency $Z=100$ or $\kappa a$ equal to 0.5 (spheres), 0.8 (pluses), and 2.1 (filled diamonds). (a) Transverse friction coefficient and; (b) longitudinal friction coefficient. In all cases, the curves are normalized by the friction coefficient of a sphere with equal surface area $\xi_{A}=6 \pi \eta \sqrt{a(a+h) / 2}$ (see Table I for the corresponding neutral values). Curves are drawn as a guide to the eye.

dence or than the effect of the particle shape. The shape effect is reflected in the substantial difference between the friction coefficients for transverse and longitudinal sedimentations. Although barely visible in Fig. 3, there is a small but significant dependence of the friction coefficients on $\kappa$. This we discuss in more detail in the next section.

The dependence on $\kappa$ is better visible in Fig. 4, where we display the friction coefficients of highly charged disks. Although here the dependence on $\kappa$ is clearly visible, it becomes less important at higher volume fractions. This suggests that with increasing volume fraction, the effect of the overlap of diffuse layers becomes less important than the direct effect of hydrodynamic interaction between disks. At small volume fractions, we always recover the $\varphi^{1 / 3}$ dependence of the inverse friction coefficients. However, deviations from this scaling are already noticeable at small $\varphi$. This fact indicates that the dilute regime is confined to smaller $\varphi$ values for highly charged disks. In the limit $\kappa a \rightarrow \infty$ (vanishing Debye length), the sedimentation friction coefficient of a charged and a neutral disk should coincide. The fact that, for finite $\kappa a$, the ratio of the friction coefficients extrapolates at $\varphi=0$ to a value different from 1 indicates the importance of electrokinetic coupling for the sedimentation of charged disks.

We stress that the values of $\kappa$ are computed on the basis of the electrolyte densities in the bulk. For concentrated suspensions of highly charged disks, the density of counterions added to the system to ensure charge neutrality $\left(\rho_{-}=-Z / V_{f}\right.$, where $Z$ is the valency of the sphere and $V_{f}$ is the volume occupied by the electrolyte) may exceed the concentration of added salt. In that case, $\boldsymbol{\kappa}^{-1}$, the screening length in the salt "reservoir" is not simply related to the apparent screening length in the dense suspension. For our simulations this phenomenon becomes important only for the highest volume fractions (typically $\varphi>0.08$ ). Hence, our extrapolation at low $\varphi$ is not affected by this complication.

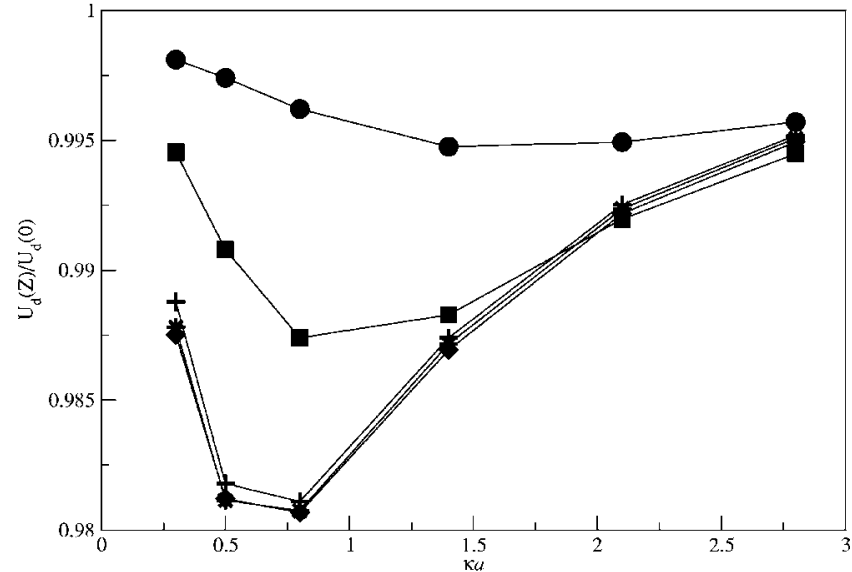

(a)

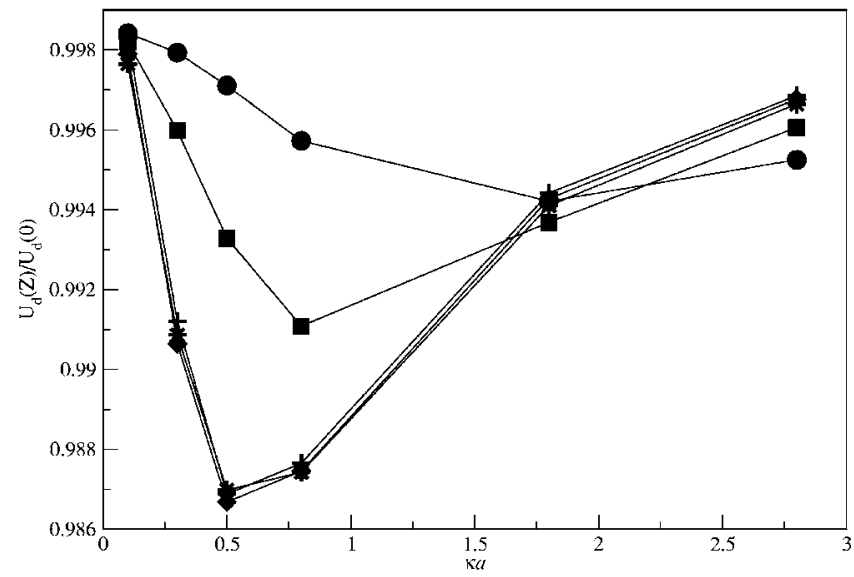

(b)

FIG. 5. Normalized sedimentation velocity of a charged disk with aspect ratio $p=5$, radius $a=5$, and valency $Z=10$ as a function of the electrical double layer (EDL) thickness expressed in dimensionless units $\kappa a$. The different simulation points correspond to $\varphi=3.8 \times 10^{-2}$ (circles), $4.8 \times 10^{-3}$ (squares), $7.3 \times 10^{-4}$ (pluses), $4.6 \times 10^{-4}$ (stars), and $3.1 \times 10^{-4}$ (diamonds). (a) Transverse motion and (b) longitudinal motion. Curves are drawn as a guide to the eye.

\section{SEDIMENTATION VELOCITY OF CHARGED DISKS: DEBYE LENGTH DEPENDENCE}

Having analyzed the role of charge and volume fraction on the sedimentation velocity, we now consider in more detail the effect of the double layer width on the sedimentation of the disks. We follow the same procedure as in Sec. III A and study the sedimentation velocity $U_{d}(Z)$ at different volume fractions, normalized by the corresponding velocity of isolated charged-neutral disks $U_{d}(0)$. In this way, the ratio $U_{d}(Z) / U_{d}(0)$ measures the reduction in the sedimentation velocity of one charged disk due to its electrokinetic interaction with the electrolyte. The reduction in sedimentation velocity in the dilute limit is interesting theoretically, because we can compare with analytic results for weakly charged spheres, although in experiments the reduced sedimentation velocity $U_{d}(Z, \varphi) / U_{d}(0, \varphi)$ at finite $\varphi$ is the relevant quantity. For simplicity, in the remaining part of this section, we will be writing $U_{d}(Z)$ instead of $U_{d}(Z, \varphi)$ but, unless explicitly stated, the volume fraction dependence is always assumed.

In Fig. 5(a), we show the normalized sedimentation velocity as a function of the double layer width for a disk with 


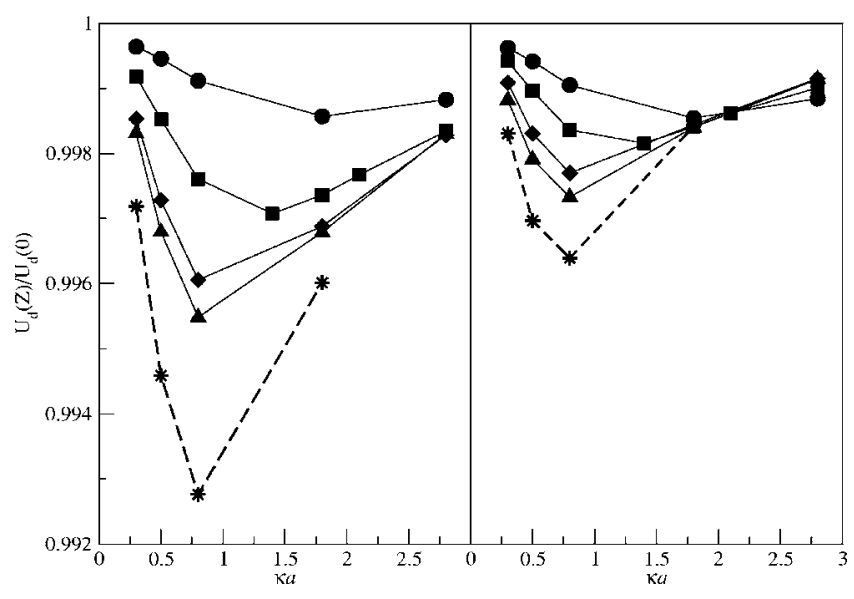

FIG. 6. Normalized sedimentation velocity of a charged disk with aspect ratio $p=10$, radius $a=10$, and valency $Z=10$ as a function of the EDL thickness expressed in dimensionless units $\kappa a$ at volume fractions $\varphi=1.9$ $\times 10^{-2}$ (circles), $\varphi=6.5 \times 10^{-3}$ (squares), $\varphi=2.9 \times 10^{-3}$ (diamonds), and $\varphi$ $=1.8 \times 10^{-3}$ (triangles), and the corresponding dilute limit extrapolation (stars). Left: Transverse motion; right: longitudinal motion. Curves are drawn as a guide to the eye.

aspect ratio $p=5$ in transverse motion, for different volume fractions. For infinitely thin and infinitely broad diffuse layers, the sedimentation velocity should coincide with that of a charged-neutral disk, and hence the curve should approach one for both small and large $\kappa a$, as is indeed observed. The decrease at intermediate values of $\kappa a$ is the result of the interplay between hydrodynamic dissipation and electrolyte diffusion. The largest effect is observed when the size of the double layer is of the order of the largest dimension of the disk, i.e., $\kappa a \sim 1$. The effect increases with decreasing volume fraction, consistent with the discussion in the previous section, and above volume fractions around $1 \%$, the changes in normalized sedimentation become negligible. The minimum velocity also depends on volume fraction, an effect which is consistent with previous findings for spheres. ${ }^{23} \mathrm{~A}$ similar behavior is observed in Fig. 5(b), where the longitudinal sedimentation for a weakly charged disk is depicted. It is interesting to note that the decrease in sedimentation velocity is slightly smaller. We can ascribe this effect to the fact that the distorted double layer is not isotropic and has a smaller contribution to the friction when the wider side of the disk is exposed to a region where the velocity gradients are smaller.

In Figs. 6(a) and 6(b), we show the sedimentation velocity for a weakly charged disk with a somewhat larger aspect ratio: $p=10$. The trends are the same as for the disk with $p$ $=5$, although the minimum velocity seems to depend on aspect ratio and is achieved now for slightly narrower double layers. The reduction in absolute terms is now smaller, but this is simply due to the lower surface charge density as compared with the smaller $(p=5)$ disk. It is worth mentioning that for disks with $p=5$ we could effectively reach the dilute limit for $U_{d}(Z) / U_{d}(0)$. In contrast, for disks with $p$ $=10$ we had to perform the dilute limit extrapolation.

In Fig. 7 we show the sedimentation velocity for a highly charged disk with $p=5$, normalized by the sedimentation velocity of uncharged disks at the same volume frac-

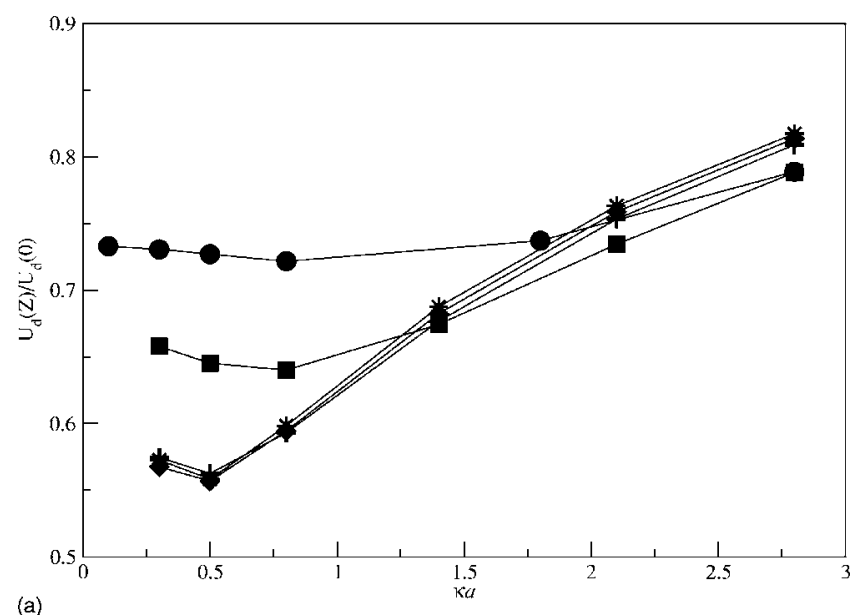

(a)

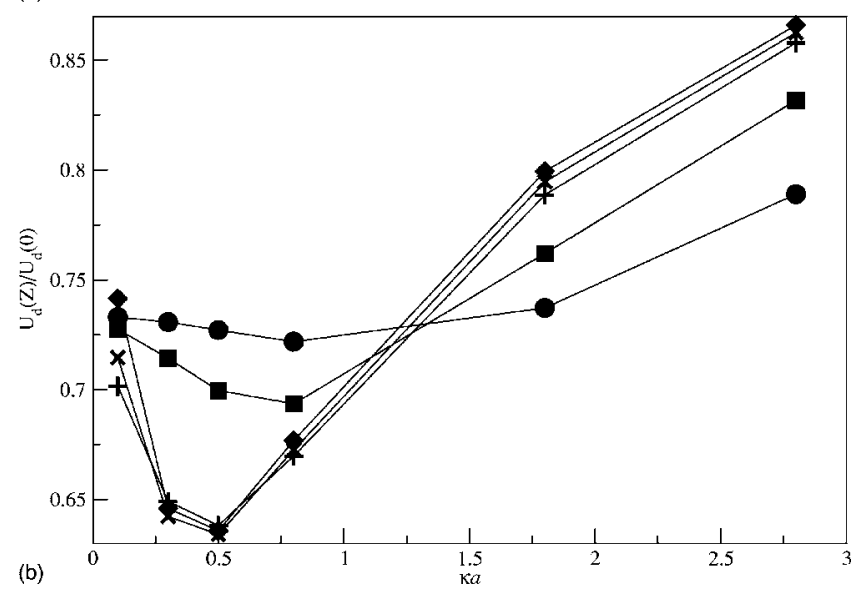

FIG. 7. Normalized sedimentation velocity of a charged disk with aspect ratio $p=5$, radius $a=5$, and valency $Z=100$ as a function of the EDL thickness expressed in dimensionless units $\kappa a$ at various volume fractions: $\varphi$ $=3.8 \times 10^{-2}$ (circles), $4.8 \times 10^{-3}$ (squares), $7.3 \times 10^{-4}$ (pluses), $4.6 \times 10^{-4}$ (stars), and $3.1 \times 10^{-4}$ (diamonds). (a) Transverse sedimentation and (b) longitudinal sedimentation. Curves are drawn as a guide to the eye.

tion. Although, again, the relevance of the electrokinetic coupling in the sedimentation velocity diminishes upon increasing volume fraction, the coupling between electric friction and velocity dissipation becomes much more dominant now. The sedimentation velocities decrease by almost $50 \%$, and the range of the values of $\kappa a$ where appreciable deviations from the uncharged disk behavior is observed is wider than in the case of weakly charged disks. Hence, the electrokinetic coupling for disks is much larger than that observed for spheres and is, in fact, consistent with mobility reductions observed in laponites. ${ }^{22}$ For higher aspect ratios the same trends are observed, as shown in Fig. 8, for the particular aspect ratio $p=10$.

In all figures we observe that the reduction in sedimentation velocity for transverse motion is larger than that for longitudinal motion. For wide double layers the differences may amount to $20 \%$. This effect can be intuitively understood in terms of the different forces felt by the electric double layer in the two configurations. For transverse sedimentation, most of the diffuse layer is exposed to the flow induced by the sedimenting array of disks. On the contrary, for longitudinal motion most of the electric double layer is located in a region where the fluid velocity is small and is 


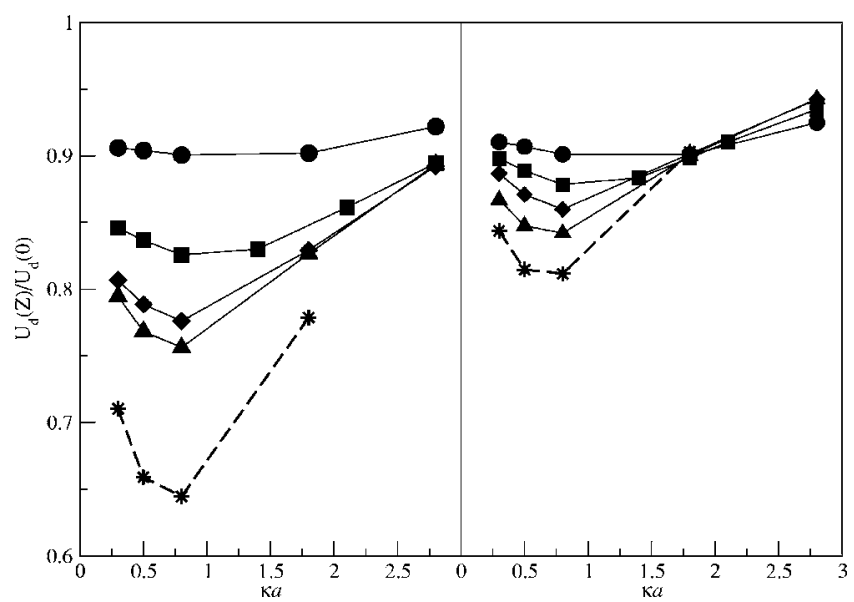

FIG. 8. Volume-fraction-dependent reduction in the sedimentation velocity of a charged disk with aspect ratio $p=10$, radius $a=10$, and valency $Z$ $=100$. The plot on the left refers to a disk sedimenting along its edge, while the right part along its symmetry axis. The different curves correspond to volume fractions $\varphi=1.9 \times 10^{-2}$ (circles), $6.5 \times 10^{-3}$ (squares), $2.9 \times 10^{-3}$ (diamonds), and $1.8 \times 10^{-3}$ (triangles), and the corresponding dilute limit extrapolation (dashed line with star symbols). Curves are drawn as a guide to the eye.

not subject to large gradients. One would then naively expect that this difference will be enhanced by an increase of the surface charge. But, in fact, the relative difference decreases with the charge of the disk. Hence, as qualitatively illustrated in Figs. 9 and 10 there are nontrivial couplings between the electrostatic restoring force and the flow field. From the figures it is clear that for longitudinal sedimentation most of the diffuse layer is in a region of smoothly varying velocity, while for transverse sedimentation the double layer is exposed to large velocity gradients. More interestingly, by comparing the flow fields past the weakly and the highly charged disk, we observe that the effective hydrodynamic shape of the particle becomes more isotropic. Moreover, close to the surface of the particle in Fig. 9(b), the direction of the flow is reversed with respect to the direction of the bulk flow. This effect can only be caused by the different behavior of the electrostatic and the hydrodynamic fields at the edge of the disks, an effect we have not analyzed in detail, because much more expensive simulations are required to gain a more quantitative understanding of the flow patterns in Fig. 9(b). Such simulations fall out of the scope of the present paper.

\section{SEDIMENTATION VELOCITY OF CHARGED DISKS: SHAPE EFFECTS}

It is not straightforward to quantify the effect of shape variations on the sedimentation of (charged) disks because one cannot change the shape without modifying either the surface charge density or the overall particle charge. Then, because the electrostatic field next to a particle is proportional to the surface charge $\sigma$, a change in the surface area will change the electric field surrounding the particle, making it impossible to isolate the effect of shape change. On the other hand, keeping $\sigma$ constant by varying the overall particle charge is not a solution either since the reduction in sedimentation velocity does depend also on $Z$ [see Eq. (5)].

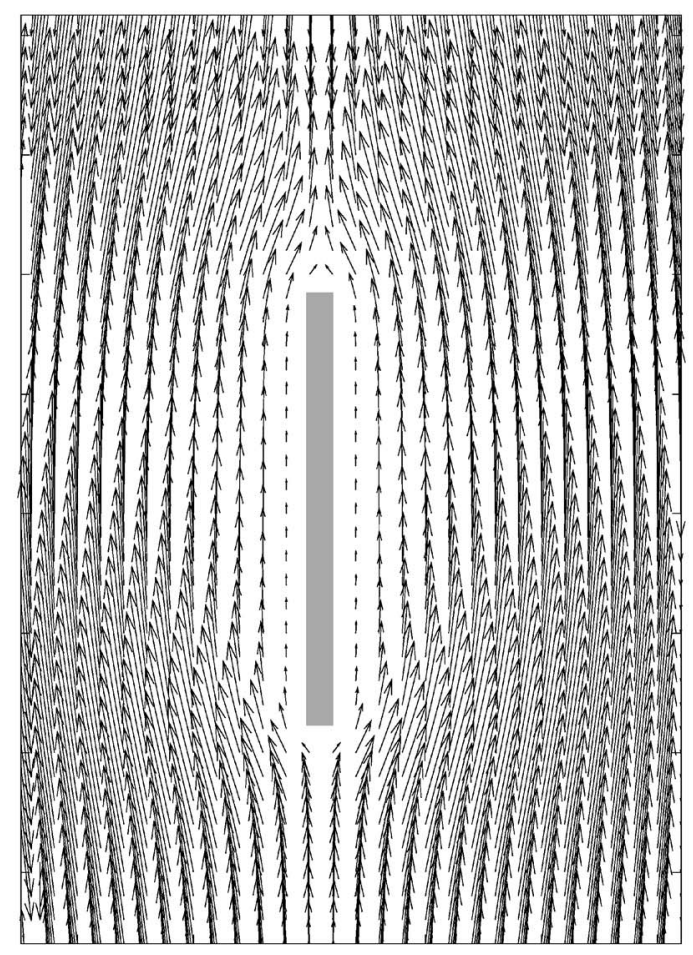

(a)

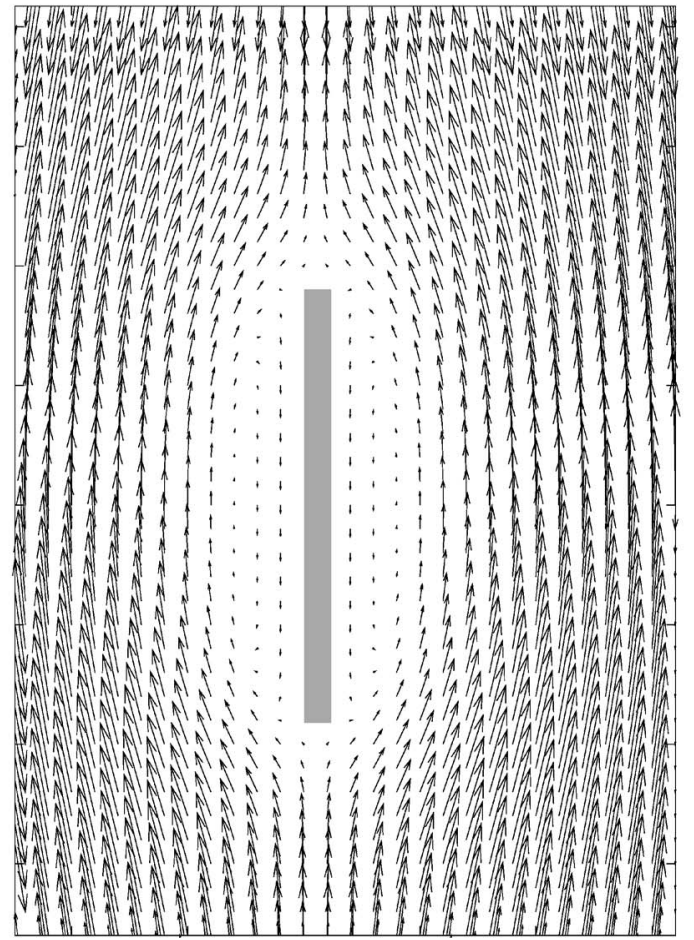

(b)

FIG. 9. Projection of the fluid velocity field near a sedimenting charged disk on the plane parallel to the axis of revolution of the disk. The disk sediment along its edge.

As a result, we will have to modify both valency and volume (to keep the surface area constant) to disentangle charge effects from effects arising from shape changes. However, even if we take care of this problem, we can only compare each disk with the corresponding sphere, because the two disks we study have different areas. 


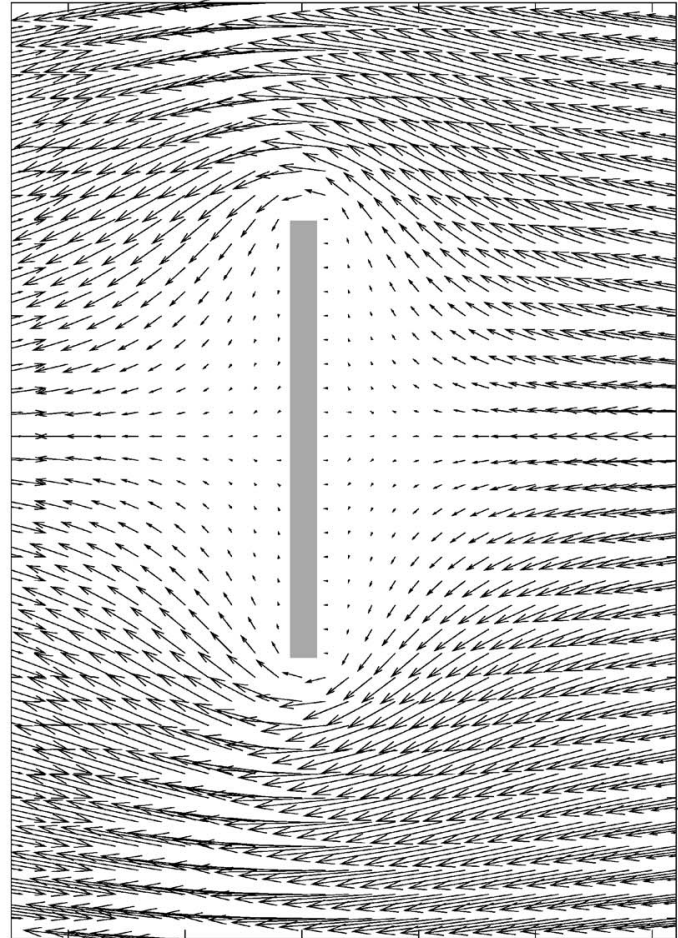

(a)

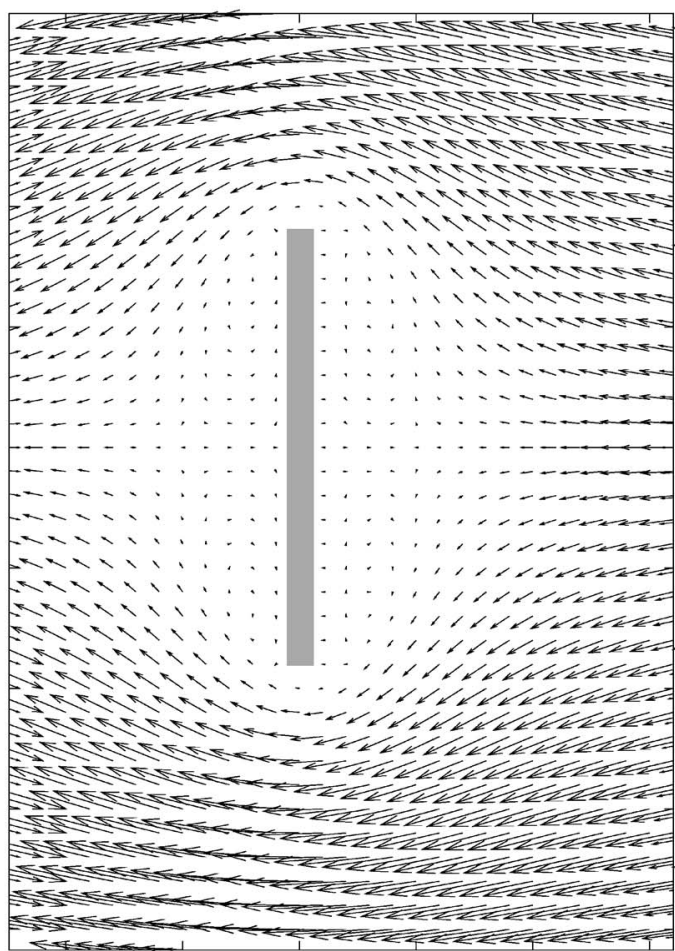

(b)

FIG. 10. Projection of the fluid velocity field near a sedimenting charged disk on the plane parallel to the axis of revolution of the disk. The disk sediment along its symmetry axis.

In order to focus on the shape effects as much as possible, we computed the normalized sedimentation velocity $U_{d}(Z) / U_{d}(0)$ [with $U_{d}(Z)$ the sedimentation velocity of an isolated particle with valency $Z$, and $U_{d}(0)$ the velocity of the same object with $Z=0$ ] with the corresponding normalized sedimentation velocity of a sphere with the same valency $Z$ and surface area, $U_{s}(Z) / U_{s}(0)$.
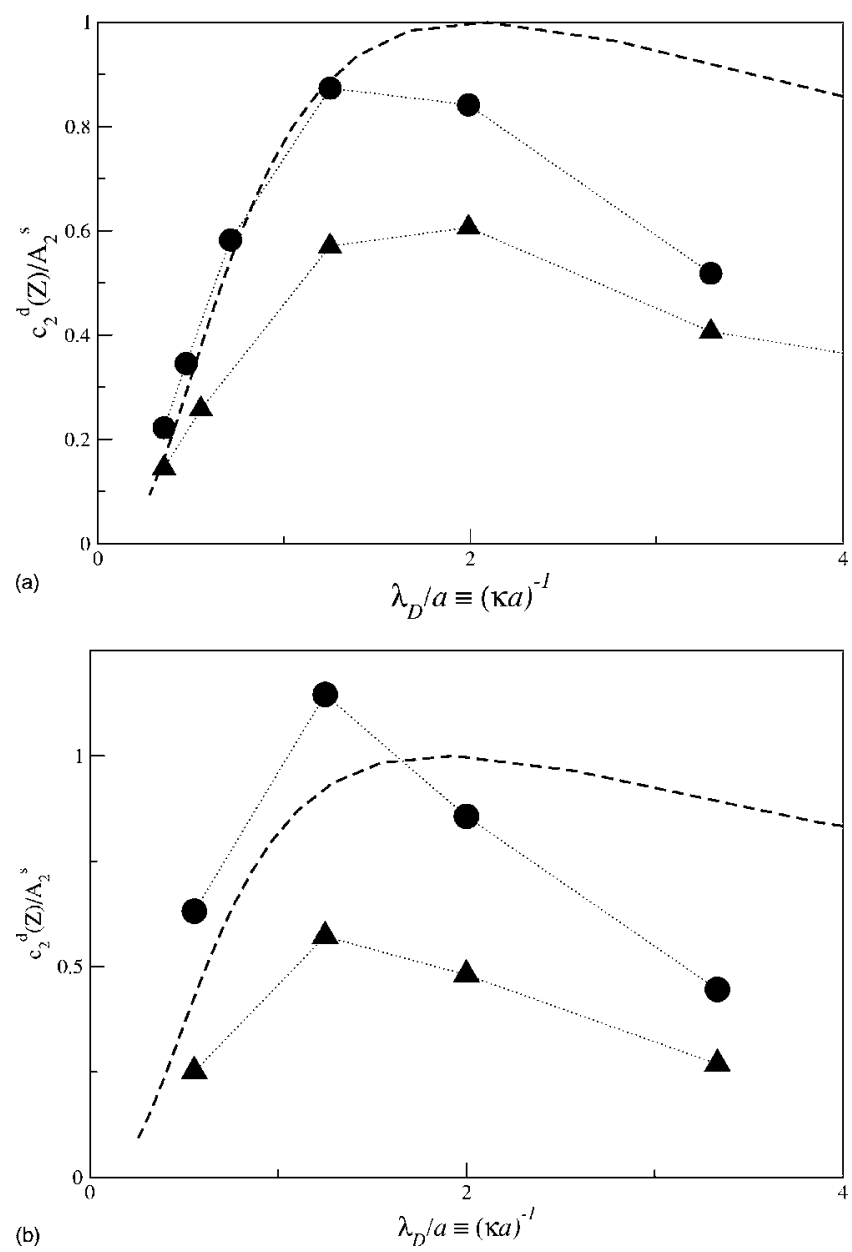

FIG. 11. Normalized reduction in the velocity of a sedimenting disk of charge $Z=10$ at infinite dilution both for transverse (spheres) and longitudinal (squares) motions as a function of its double layer thickness. The dashed curve corresponds to the theoretical prediction for a sedimenting sphere of equal charge and surface area. (a) $p=5$ and (b) $p=10$. Lines joining the simulation points are drawn as a guide to the eye.

For weakly charged particles, we can make use of Booth's prediction to analyze the results. To this end, rather than studying the scaled velocity directly, we have found fruitful to consider $\left[1-U_{d}(Z) / U_{d}(0)\right] / Z^{2}$, which is the coefficient $c_{2}$ [see Eq. (6)] in the case of a sphere. This is a direct measure of the electrokinetic reaction induced by the electric double layer. Since we have argued (see Sec. IV) that the charge dependence of disks is the same as the one observed for spheres in the Debye-Hückel limit, the previous ratio is a quantitative way of assessing the role of shape on the sedimentation velocity.

In Figs. 11(a) and 11(b), we show [1 $\left.-U_{d}(Z) / U_{d}(0)\right] / Z^{2} \equiv c_{2}^{d}$ for disks with two different aspect ratios and with a small charge, $Z=10$, both for transverse and longitudinal sedimentations. $c_{s}^{d}$ is expressed in units of $A_{2}^{s}$ $\equiv k_{B} T l_{B} /\left(72 \pi D \eta a^{2}\right)$, in such a way that for spheres it reduces to $f(\kappa a)$ as predicted by Booth. For weakly charged disks and thin double layers, the decrease in velocity does not depend strongly on shape. This is consistent with Smoluchowski's theory for electrophoresis, ${ }^{24}$ which predicts that the electrophoretic velocity of particles with the same zeta potential (the electrostatic potential at contact) is indepen- 

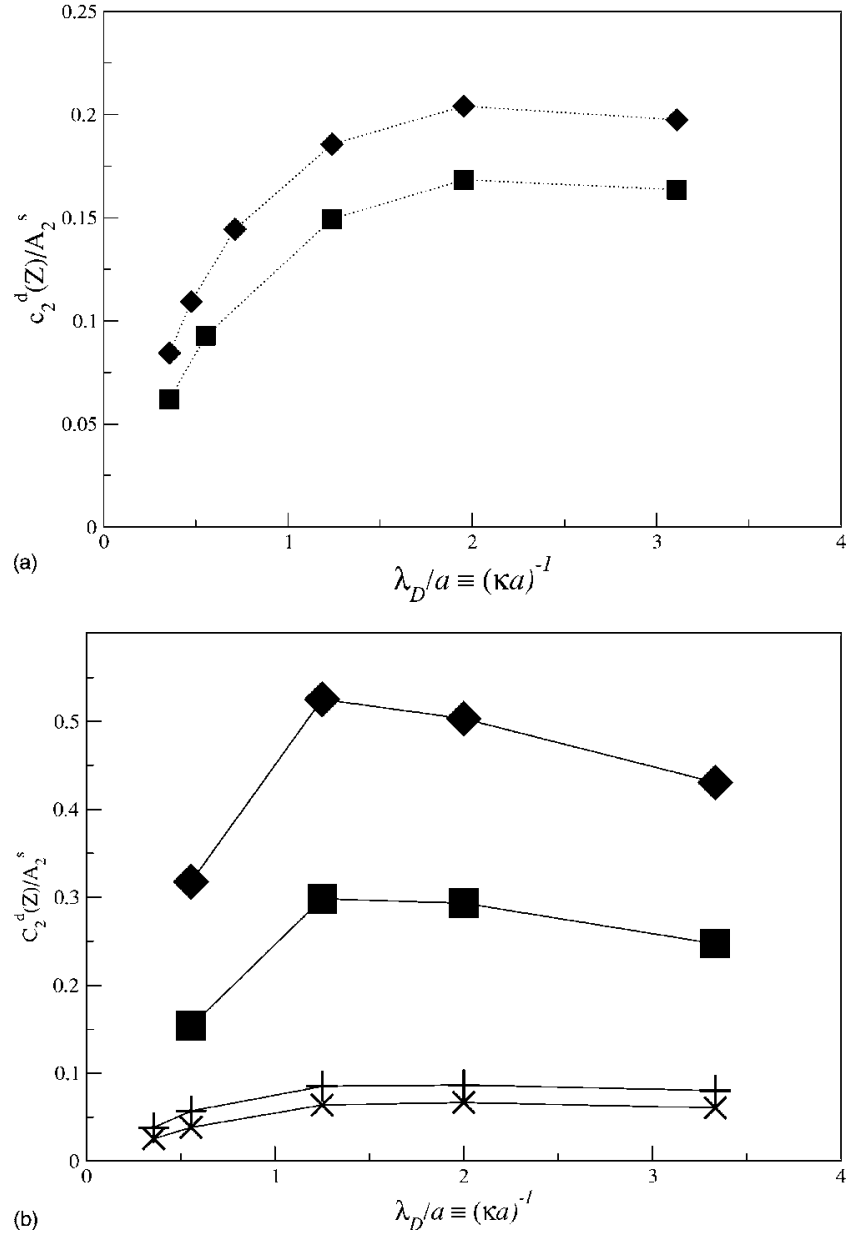

FIG. 12. Normalized reduction in the velocity of a sedimenting highly charged disk at infinite dilution both for transverse (spheres) and longitudinal (squares) motions as a function of its double layer thickness. The dashed curve corresponds to the theoretical prediction for a sedimenting sphere of equal charge and surface area. (a) $p=5$ and (b) $p=10$. Lines joining the simulation points are drawn as a guide to the eye. Disks and spheres have a valency $Z=100$, which correspond to a surface charge $\sigma=0.45$ for the disk with $p=5$ and to $\sigma=0.13$ for the disk with $p=10$. In subfigure (b) we also show the same simulation for a disk surface charge $\sigma=0.40$ for the disk with $p=10$ in transverse $(+)$ and longitudinal $(\times)$ sedimentations. Lines joining the simulation points are drawn as a guide to the eye.

dent of the particle shape if $\kappa a \rightarrow \infty$. However, the deviation from this Smoluchowski limit appears confined at narrower double layers for longitudinal motion; hence, the shape affects significantly the sedimentation velocity of suspended particles. Moreover, in the case of asymmetric objects, the orientation of the particle also affects the velocity. For both longitudinal and transverse sedimentations, the electrokinetic coupling of a disk is always smaller than the decrease for an equivalent sphere. One can clearly see that the decrease in velocity for longitudinal motion is smaller than for transverse motion.

In the high-charge regime we use the same quantity, $c_{2}^{d}$, to assess the role of shape, although we know that the Booth theory fails in this case. In Figs. 12(a) and 12(b) we show $c_{2}^{d}$, again for two aspect ratios. In the thin diffuse-layer limit, our data are consistent with Smoluchowski's theory, and we observe again a departure from the results for a sphere upon increasing the width of the electric double layer. The maxi-

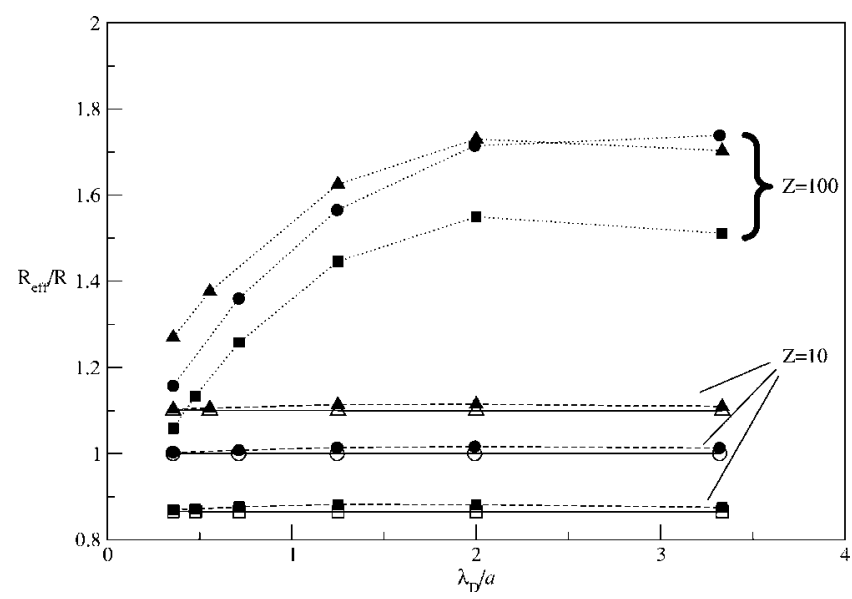

FIG. 13. Effective hydrodynamic radius of a disk normalized by the radius $R$ of a sphere with equivalent surface as a function of the inverse Debye length in units of the disk radius. Disk with $p=5$ in transverse (square) and longitudinal (triangle) motions; sphere with the same surface (circle). Uncharged objects (drawn line and open symbols), $Z=10$ (dashed curve), and $Z=100$ (dotted line). The curves are drawn as a guide to the eye.

mum effect is observed for electric-double-layer widths of the order of the largest linear dimension of the object, and, again, the decrease for longitudinal motion is smaller than for transverse motion. This is consistent with the intuitive picture that the hydrodynamic shape of a disk becomes more isotropic upon increasing its charge.

By comparing Figs. 12(a) and 12(b), the reader might conclude that the reduction in sedimentation velocity is higher for the disk with a smaller aspect ratio. However, one should bear in mind that the two disks have the same valency and therefore very different surface charges $\sigma_{p=5} \simeq 3.4$ $\times \sigma_{p=10}$. To show how much the surface charge affects $c_{2}$, we show $c_{2}$ for the disk with $p=10$ also at $Z=300$. Even though the surface charge of this disk is still lower than the surface charge for the other disk, the electrokinetic effect is already more pronounced.

An illuminating way of displaying the relevance of shape for sedimentation is to consider what the effective Stokes radius of a sedimenting disk is. In Fig. 13 we show the effective Stokes radius $\left[R_{\text {eff }} \equiv F /(6 \pi \eta U)\right]$, where $F$ is the magnitude of the external force acting on the disk. For small charges, the effective radius depends weakly on the width of the double layer and is larger for the transverse motion, as can be expected. At high charges the behavior is qualitatively different since $R_{\text {eff }}$ depends on the width of the double layer for $\lambda_{D} / a<2$. For larger $\lambda_{D} / a$ it tends to level off. As the diffuse layer broadens, the effective size that characterizes the sphere and the disk in longitudinal motion tend to converge, leading to a same effective shape for wide layers.

The physical origin of this effect is already implicit in Figs. 9 and 10. These figures show the velocity fields around the disk for both orientations. Different flow fields develop around the sedimenting disk for low and high surface charges. The flow profiles look more isotropic for high $Z$; therefore, one might expect that for high $Z$, the friction coefficients of a disk approach that of a sphere with the same $Z$. 


\section{DISCUSSION}

In this paper we have presented simulations of the sedimentation of an array of charged disks. We have treated the electrolyte at the Poisson-Boltzmann level, while we have incorporated the relevant hydrodynamic couplings between the solvent and the dissolved electrolyte. Using the latticeBoltzmann method we have modeled highly charged colloids and arbitrary $\kappa a$ values, which greatly expands the parameter range that can be covered.

Since no exact analytical expressions exist for the sedimentation velocity of isolated neutral disks and finite thickness, we have first checked the performance of our method by validating the sedimentation of neutral disks using approximate expressions that become exact for infinitesimally thin disks. Such a computation has provided us with values for the sedimentation velocities of uncharged disks, which are needed for the subsequent analysis.

In order to clarify the role of electrohydrodynamic coupling and the relevance of shape, we have performed a systematic study to assess the role of shape, volume fraction, charge, and ionic strength on the sedimentation velocity. We find that in the linearized Debye-Hückel regime, the sedimentation velocity has the same functional dependence on volume fraction and surface charge as that for spheres, although with different amplitudes. This deviation should be accounted for when using diffusivity measurements of disks to infer the effective charge of colloids, and this work represents, to our knowledge, the first results where the sedimentation velocity is computed systematically. So far experimental findings could only be compared with the theory for weakly charged spheres, ${ }^{22}$ which can lead to numerical errors in the estimates of their effective charges. At fixed $\kappa a$, we have studied the surface charge dependence of the disk sedimentation velocity, from which we have observed that in the high-charge regime, the accumulation of charge near the disk surface layer decreases the effect of electrokinetic coupling on the sedimentation velocity and also shows that such accumulation becomes more relevant as the disk becomes more anisotropic.

We have shown that the geometrical anisotropies of neutral disks are reduced by the presence of the electric double layer, especially for highly charged disks. In fact, we have seen that when the double layer is exposed to larger veloci- ties, the reduction in sedimentation velocity is larger. Hence, this mechanism tends to generate a more symmetric disk response, as can be effectively characterized in terms of an effective disk radius which becomes less sensitive to shape details as the charge increases.

\section{ACKNOWLEDGMENTS}

The authors acknowledge Professor M. Fixman for his insightful comments. The work of the FOM Institute is part of the research program of FOM and is made possible by financial support from the Netherlands Organization for Scientific Research (NWO). One of the authors (I.P.) thanks DGICYT of the Spanish Government, Distinció de la Generalitat de Catalunya (Spain) for financial support, and the FOM Institute for its hospitality.

${ }^{1}$ A. P. Philipse, Curr. Opin. Colloid Interface Sci. 2, 200 (1997).

${ }^{2}$ A. Ortega and J. G. de la Torre, J. Chem. Phys. 119, 9914 (2003).

${ }^{3}$ S. Kutter, J. P. Hansen, M. Sprik, and E. Boek, J. Chem. Phys. 112, 311 (2000).

${ }^{4}$ G. Odriozola, M. Romero-Bastida, and F. D. Guevara-Rodriguez, Phys. Rev. E 70, 021405 (2004).

${ }^{5}$ D. Long, J. L. Viovy, and A. Ajdari, Phys. Rev. Lett. 76, 3858 (1996).

${ }^{6}$ D. Long and A. Ajdari, Phys. Rev. Lett. 81, 1529 (1998).

${ }^{7}$ A. Chatterji and J. Horbach, J. Chem. Phys. 122, 184903 (2005).

${ }^{8}$ V. Lobaskin and B. Düweg, J. Phys.: Condens. Matter 16, S4063 (2004).

${ }^{9}$ S. Melchionna and S. Succi, J. Chem. Phys. 120, 4492 (2004).

${ }^{10}$ Z. L. Guo, T. S. Zhao, and Y. Shi, J. Chem. Phys. 122, 144907 (2005).

${ }^{11}$ H. Kodama, K. Takeshista, T. Araki, and H. Tanaka, J. Phys.: Condens. Matter 16, L115 (2004).

${ }^{12}$ F. Capuani, I. Pagonabarraga, and D. Frenkel, J. Chem. Phys. 121, 973 (2004).

${ }^{13}$ R. J. Hunter, Foundations of Colloid Science (Oxford Science, Oxford, (1989).

${ }^{14}$ N. Rossi, S. Ubertini, G. Bella, and S. Succi, Int. J. Numer. Methods Fluids 49, 619 (2005).

${ }^{15}$ A. J. C. Ladd, J. Fluid Mech. 271, 285 (1994).

${ }^{16}$ M. E. Cates, J. C. Desplat, P. Stansell, A. J. Wagner, K. Stratford, R. Adhikari, and I. Pagonabarraga, Philos. Trans. R. Soc. London, Ser. A 363, 1917 (2005).

${ }^{17}$ W. E. Williams, J. Fluid Mech. 25, 589 (1966).

${ }^{18}$ J. Happel and H. H. Brenner, Low Reynolds Number Hydrodynamics (Nijhoff, Dordrecht, The Netherlands, (1983).

${ }^{19}$ H. Hashimoto, J. Fluid Mech. 5, 317 (1959).

${ }^{20}$ F. Booth, J. Chem. Phys. 22, 1956 (1954).

${ }^{21}$ H. Ohshima, T. W. Healy, L. R. White, and R. W. O'Brien, J. Chem. Soc., Faraday Trans. 2 80, 1299 (1982).

${ }^{22}$ S. L. Tawari, D. L. Koch, and C. Cohen, J. Colloid Interface Sci. 240, 54 (2001).

${ }^{23}$ H. J. Keh and J. M. Ding, J. Colloid Interface Sci. 227, 540 (2000).

${ }^{24}$ M. von Smoluchowski, Phys. Chem. 92, 129 (1918). 